\title{
A new hybrid algorithm and its numerical realization for two nonexpansive mappings
}

\author{
Qiao-Li Dong ${ }^{1,2}$, Songnian $\mathrm{He}^{1,2}$ and Yeol Je Cho $\mathrm{CH}^{34^{*}}$
}

\footnotetext{
"Correspondence: yjcho@gnu.ac.kr ${ }^{3}$ Department of Mathematics Education and RINS, Gyeongsang National University, Jinju, 660-701, Korea

${ }^{4}$ Department of Mathematics, Faculty of Science, King Abdulaziz University, Jeddah, Saudi Arabia Full list of author information is available at the end of the article
}

\begin{abstract}
In the paper, first, we introduce a new hybrid projection algorithm and present its strong convergence theorem. Next, we analyze different hybrid algorithms in computing and conclude that our proposed algorithm has an advantage. Finally, the numerical experiments validate the efficiency and advantages of the new algorithm.
\end{abstract}

MSC: $90 C 47 ; 49 J 35$

Keywords: nonexpansive mapping; hybrid algorithm; cyclic algorithm; parallel algorithm; strong convergence

\section{Introduction}

Let $H$ be a real Hilbert space with the inner product $\langle\cdot, \cdot\rangle$ and the norm $\|\cdot\|$ and $C$ be a nonempty closed convex subset of $H$. Recall that a mapping $T: C \rightarrow C$ is said to be nonexpansive if

$$
\|T x-T y\| \leq\|x-y\|
$$

for all $x, y \in C$. We denote by $\operatorname{Fix}(T)$ the set of fixed points of $T$, i.e., $\operatorname{Fix}(T)=\{x \in C: T x=$ $x\}$.

The construction of common fixed points for a finite family of nonlinear mappings is of practical importance. In particular, iteration algorithms for finding common fixed points of a finite family of nonexpansive mappings have received extensive investigation (see [13]) since these algorithms have a variety of applications in inverse problem, image recovery, and signal processing (see [4-7]).

Mann's iteration algorithm [8] is often used to find a fixed point of nonexpansive mappings, but it has only weak convergence (see [9] for an example). However, strong convergence is often much more desirable than weak convergence in many problems that arise in infinite dimensional spaces (see [10] and references therein). So, attempts have been made to modify Mann's iteration algorithm so that strong convergence is guaranteed. Let $T: C \rightarrow C$ be a nonexpansive mapping. Then $I-T$ is a maximal monotone operator [11]. Inspired by Solodov and Svaiter's hybrid method for finding a zero of a maximal monotone operator [12], Nakajo and Takahashi [13] first introduced a hybrid algorithm for a nonexpansive mapping. Thereafter, some hybrid algorithms have been studied extensively since they have strong convergence (see [14-18]).

(c) 2015 Dong et al. This article is distributed under the terms of the Creative Commons Attribution 4.0 International License (http://creativecommons.org/licenses/by/4.0/), which permits unrestricted use, distribution, and reproduction in any medium, provided you give appropriate credit to the original author(s) and the source, provide a link to the Creative Commons license, and indicate if changes were made. 
In this paper, motivated by Eckstein and Svaiter's splitting methods for approximating a zero of the sum of two maximal monotone operators [19], we introduce a new hybrid algorithm. Let $T, S: C \rightarrow C$ be two nonexpansive mappings such that $\operatorname{Fix}(T) \cap \operatorname{Fix}(S) \neq \emptyset$. We consider the following algorithm.

\section{Algorithm 1}

$$
\left\{\begin{array}{l}
x_{0} \in C \text { chosen arbitrarily, } \\
y_{n}=\alpha_{n} x_{n}+\left(1-\alpha_{n}\right) T x_{n}, \\
z_{n}=\beta_{n}\left[\gamma_{n} y_{n}+\left(1-\gamma_{n}\right) x_{n}\right]+\left(1-\beta_{n}\right) S y_{n}, \\
C_{n}=\left\{z \in C: \sigma\left\|z_{n}-z\right\|^{2}+(1-\sigma)\left\|y_{n}-z\right\|^{2} \leq\left\|x_{n}-z\right\|^{2}\right\} \\
Q_{n}=\left\{z \in C:\left\langle x_{n}-z, x_{n}-x_{0}\right\rangle \leq 0\right\} \\
x_{n+1}=P_{C_{n} \cap Q_{n}} x_{0}
\end{array}\right.
$$

for each $n \geq 0$, where $P_{K}$ denotes the metric projection onto the set $K$ and $\alpha_{n}, \beta_{n} \in[0,1-$ $\delta], \delta \in[0,1), \gamma_{n} \in[0,1], \sigma \in(0,1)$ with some conditions.

\section{Relation to the previous work}

In this section, we analyze and compare Algorithm 1 with two important hybrid algorithms which are simple and easily realized. For the purposes of comparison, set $\gamma_{n}=1$ in Algorithm 1, which is reasonable from the numerical experiments in Section 5. In the case where $\gamma_{n}=1$, Algorithm 1 actually is a modification of the following cyclic algorithm:

$$
\left\{\begin{array}{l}
y_{n}=\alpha_{n} x_{n}+\left(1-\alpha_{n}\right) T x_{n} \\
x_{n+1}=\beta_{n} y_{n}+\left(1-\beta_{n}\right) S y_{n}
\end{array}\right.
$$

for each $n \geq 0$, where $x_{0} \in C$.

In [20], Takahashi et al. modified the cyclic algorithm (2) and introduced another hybrid algorithm.

\section{Algorithm 2}

$$
\left\{\begin{array}{l}
x_{0} \in C \text { chosen arbitrarily, } \\
y_{n}=\alpha_{n} x_{n}+\left(1-\alpha_{n}\right) T_{[n]} x_{n} \\
C_{n}=\left\{z \in C:\left\|y_{n}-z\right\| \leq\left\|x_{n}-z\right\|\right\} \\
Q_{n}=\left\{z \in C:\left\langle x_{n}-z, x_{n}-x_{0}\right\rangle \leq 0\right\} \\
x_{n+1}=P_{C_{n} \cap Q_{n}} x_{0}
\end{array}\right.
$$

for each $n \geq 0$, where $0 \leq \alpha_{n} \leq \alpha<1, T_{[n]}=T_{n} \bmod 2$ and the $\bmod$ function takes values in $\{1,2\}$ and $T_{1}=T$ and $T_{2}=S$.

The computational complexity of Algorithm 1 on every step is one computation of a metric projection and two values of $S$ and $T$, while the computational complexity of Algorithm 2 on every step is one computation of a metric projection and one value of $S$ or $T$. In general, the computational cost of metric projection is larger than that of operators. 
By modifying the parallel algorithm (see [4]), it is easy to obtain the following algorithm (see [21] for details).

\section{Algorithm 3}

$$
\left\{\begin{array}{l}
x_{0} \in C \text { chosen arbitrarily, } \\
y_{n}=\alpha_{n} x_{n}+\beta_{n} T x_{n}+\gamma_{n} S x_{n}, \\
C_{n}=\left\{z \in C:\left\|y_{n}-z\right\| \leq\left\|x_{n}-z\right\|\right\}, \\
Q_{n}=\left\{z \in C:\left\langle x_{n}-z, x_{n}-x_{0}\right\rangle \leq 0\right\}, \\
x_{n+1}=P_{C_{n} \cap Q_{n}} x_{0}
\end{array}\right.
$$

for each $n \geq 0$, where $\alpha_{n}, \beta_{n}, \gamma_{n} \in[0,1], \alpha_{n}+\beta_{n}+\gamma_{n}=1$ and $\lim _{\sup _{n \rightarrow \infty}} \alpha_{n}<1$, $\liminf _{n \rightarrow \infty} \beta_{n} \gamma_{n}>0$.

In the sense of computational complexity Algorithm 3 is similar to Algorithm 1. However, it is generally recognized that the cyclic algorithm (like the Gauss-Seidel iteration) is faster than the parallel algorithm (like Jacob iteration).

\section{Preliminaries}

We use the following notation:

- $\rightarrow$ for weak convergence and $\rightarrow$ for strong convergence;

- $\omega_{w}\left(x_{n}\right)=\left\{x: \exists x_{n_{j}} \rightarrow x\right\}$ denotes the weak $\omega$-limit set of $\left\{x_{n}\right\}$.

We need some facts and tools in a real Hilbert space $H$ which are listed as lemmas below.

Lemma 3.1 We have the identity in a real Hilbert space $H$ :

$$
\|u-v\|^{2}=\|u\|^{2}-\|v\|^{2}-2\langle u-v, v\rangle
$$

for all $u, v \in H$.

Lemma 3.2 (Goebel and Kirk [22]) Let $C$ be a nonempty closed convex subset of a real Hilbert space $H$ and $T: C \rightarrow C$ be a nonexpansive mapping such that $\operatorname{Fix}(T) \neq \emptyset$. If a sequence $\left\{x_{n}\right\}$ in $C$ is such that $x_{n} \rightarrow z$ and $x_{n}-T x_{n} \rightarrow 0$, then $z=T z$.

Lemma 3.3 Let $C$ be a nonempty closed convex subset of real Hilbert space $H$ and let $P_{C}$ be the (metric or nearest point) projection from $H$ onto $C$ (i.e., for any $x \in H, P_{C} x$ is the only point in $C$ such that $\left.\left\|x-P_{C} x\right\|=\inf \{\|x-z\|: z \in C\}\right)$. Then, for any $x \in H$ and $z \in C$, $z=P_{C} x$ if and only if

$$
\langle x-z, y-z\rangle \leq 0
$$

for all $y \in C$.

Lemma 3.4 (Martinez-Yanes and Xu [23]) Let $C$ be a nonempty closed convex subset of $H$. Let $\left\{x_{n}\right\}$ be a sequence in $H$ and $u \in H$. Let $q=P_{C} u$. If $\left\{x_{n}\right\}$ is such that $\omega_{w}\left\{x_{n}\right\} \subset C$ and 
satisfies the condition

$$
\left\|x_{n}-u\right\| \leq\|u-q\|
$$

for all $n \geq 1$, then $x_{n} \rightarrow q$.

Lemma 3.5 Let $H$ be a real Hilbert space. Let $C$ be a nonempty closed convex subset of $H$ and let $x, y, z \in H$. For any real number $\sigma \in[0,1]$, the set

$$
D:=\left\{v \in C: \sigma\|z-v\|^{2}+(1-\sigma)\|y-v\|^{2} \leq\|x-v\|^{2}\right\}
$$

is convex (and closed).

Proof In fact, the defining inequality in $D$ is equivalent to the inequality

$$
\langle\sigma(z-x)+(1-\sigma)(y-x), x-v\rangle \leq-\frac{1}{2}\left(\sigma\|z-x\|^{2}+(1-\sigma)\|y-x\|^{2}\right) .
$$

This inequality is affine in $v$ and hence the set $D$ is convex.

\section{Main results}

In this section, we first present a strong convergence theorem and its proof for Algorithm 1. Then we extend it to a finite family of nonexpansive mappings.

Theorem 4.1 Let $C$ be a nonempty closed convex subset of a Hilbert space $H$ and $T, S$ : $C \rightarrow C$ be two nonexpansive mappings such that $\operatorname{Fix}(T) \cap \operatorname{Fix}(S) \neq \emptyset$. Assume that $\left\{\alpha_{n}\right\}$, $\left\{\beta_{n}\right\}$, and $\left\{\gamma_{n}\right\}$ are the sequences in $[0,1]$ such that $\alpha_{n}, \beta_{n} \leq 1-\delta$ for some $\delta \in(0,1]$. Assume $\sigma \in(0,1)$. Then the sequence $\left\{x_{n}\right\}$ generated by Algorithm 1 converges in norm to $P_{\operatorname{Fix}(T) \cap \mathrm{Fix}(S) x_{0}}$.

Proof First, observe that $C_{n}$ is convex by Lemma 3.5. Next, we show that $\operatorname{Fix}(T) \cap \operatorname{Fix}(S) \subset$ $C_{n}$ for all $n \geq 0$. To observe this, arbitrarily take $p \in \operatorname{Fix}(T) \cap \operatorname{Fix}(S)$, and we have

$$
\left\|y_{n}-p\right\| \leq \alpha_{n}\left\|x_{n}-p\right\|+\left(1-\alpha_{n}\right)\left\|T x_{n}-p\right\| \leq\left\|x_{n}-p\right\|
$$

and

$$
\begin{aligned}
\left\|z_{n}-p\right\| & \leq \beta_{n}\left[\gamma_{n}\left\|y_{n}-p\right\|+\left(1-\gamma_{n}\right)\left\|x_{n}-p\right\|\right]+\left(1-\beta_{n}\right)\left\|S y_{n}-p\right\| \\
& \leq \beta_{n}\left\|x_{n}-p\right\|+\left(1-\beta_{n}\right)\left\|y_{n}-p\right\| \\
& \leq\left\|x_{n}-p\right\| .
\end{aligned}
$$

Combining the above inequalities, it follows that, for any $\sigma \in(0,1)$,

$$
\sigma\left\|z_{n}-p\right\|^{2}+(1-\sigma)\left\|y_{n}-p\right\|^{2} \leq\left\|x_{n}-p\right\|^{2},
$$

and so $p \in C_{n}$ for all $n \geq 0$. 
Next, we show that

$$
\operatorname{Fix}(T) \cap \operatorname{Fix}(S) \subset Q_{n}
$$

for all $n \geq 0$ by induction. For $n=0$, we have $\operatorname{Fix}(T) \cap \operatorname{Fix}(S) \subset C=Q_{0}$. Assume that $\operatorname{Fix}(T) \cap \operatorname{Fix}(S) \subset Q_{n}$. Since $x_{n+1}$ is the projection of $x_{0}$ onto $C_{n} \cap Q_{n}$, by Lemma 3.3, we have

$$
\left\langle x_{n+1}-z, x_{n+1}-x_{0}\right\rangle \leq 0
$$

for all $z \in C_{n} \cap Q_{n}$. Since $\operatorname{Fix}(T) \cap \operatorname{Fix}(S) \subset C_{n} \cap Q_{n}$, by the induction assumption, the last inequality holds, in particular, for all $z \in \operatorname{Fix}(T) \cap \operatorname{Fix}(S)$. This together with the definition of $Q_{n+1}$ implies that $\operatorname{Fix}(T) \cap \operatorname{Fix}(S) \subset Q_{n+1}$. Hence (3) holds for all $n \geq 0$.

Now, since $x_{n}=P_{Q_{n}} x_{0}$ (by the definition of $Q_{n}$ ) and $\operatorname{Fix}(T) \cap \operatorname{Fix}(S) \subset Q_{n}$, we have

$$
\left\|x_{n}-x_{0}\right\| \leq\left\|p-x_{0}\right\|
$$

for all $p \in \operatorname{Fix}(T) \cap \operatorname{Fix}(S)$. In particular, $\left\{x_{n}\right\}$ is bounded and

$$
\left\|x_{n}-x_{0}\right\| \leq\left\|q-x_{0}\right\|
$$

where $q=P_{\mathrm{Fix}(T) \cap \mathrm{Fix}(S)} x_{0}$. The fact that $x_{n+1} \in Q_{n}$ implies that $\left\langle x_{n+1}-x_{n}, x_{n}-x_{0}\right\rangle \geq 0$. This together with Lemma 3.1 implies that

$$
\begin{aligned}
\left\|x_{n+1}-x_{n}\right\|^{2} & =\left\|\left(x_{n+1}-x_{0}\right)-\left(x_{n}-x_{0}\right)\right\|^{2} \\
& =\left\|x_{n+1}-x_{0}\right\|^{2}-\left\|x_{n}-x_{0}\right\|^{2}-2\left\langle x_{n+1}-x_{n}, x_{n}-x_{0}\right\rangle \\
& \leq\left\|x_{n+1}-x_{0}\right\|^{2}-\left\|x_{n}-x_{0}\right\|^{2},
\end{aligned}
$$

which implies

$$
\left\|x_{n+1}-x_{n}\right\| \rightarrow 0
$$

as $n \rightarrow \infty$. Observe that $x_{n+1} \in C_{n}$ implies that

$$
\sigma\left\|z_{n}-x_{n+1}\right\|^{2}+(1-\sigma)\left\|y_{n}-x_{n+1}\right\|^{2} \leq\left\|x_{n}-x_{n+1}\right\|^{2} .
$$

Due to $\sigma \in(0,1)$, we have

$$
\left\|z_{n}-x_{n+1}\right\| \rightarrow 0, \quad\left\|y_{n}-x_{n+1}\right\| \rightarrow 0
$$

as $n \rightarrow \infty$, which yields

$$
\left\|z_{n}-y_{n}\right\| \rightarrow 0
$$

Combining (5) and (6), we obtain

$$
\left\|z_{n}-x_{n}\right\| \rightarrow 0, \quad\left\|y_{n}-x_{n}\right\| \rightarrow 0
$$


as $n \rightarrow \infty$. Noting that $\left(1-\alpha_{n}\right)\left(T x_{n}-x_{n}\right)=y_{n}-x_{n}$, we obtain

$$
\left\|T x_{n}-x_{n}\right\|=\frac{1}{1-\alpha_{n}}\left\|y_{n}-x_{n}\right\| .
$$

Since $\alpha_{n} \leq 1-\delta$, by (8), we have

$$
\left\|T x_{n}-x_{n}\right\| \rightarrow 0
$$

as $n \rightarrow \infty$. From $z_{n}=\beta_{n}\left[\gamma_{n} y_{n}+\left(1-\gamma_{n}\right) x_{n}\right]+\left(1-\beta_{n}\right) S y_{n}$, we have

$$
\begin{aligned}
\left\|S y_{n}-z_{n}\right\| & =\frac{\beta_{n}}{1-\beta_{n}}\left\|\gamma_{n}\left(y_{n}-z_{n}\right)+\left(1-\gamma_{n}\right)\left(x_{n}-z_{n}\right)\right\| \\
& \leq \frac{1}{1-\beta_{n}}\left(\gamma_{n}\left\|y_{n}-z_{n}\right\|+\left(1-\gamma_{n}\right)\left\|x_{n}-z_{n}\right\|\right),
\end{aligned}
$$

which, from (7)-(8), yields

$$
\left\|S y_{n}-z_{n}\right\| \rightarrow 0
$$

as $n \rightarrow \infty$ and so

$$
\begin{aligned}
\left\|S x_{n}-x_{n}\right\| & \leq\left\|S x_{n}-S y_{n}\right\|+\left\|S y_{n}-z_{n}\right\|+\left\|z_{n}-x_{n}\right\| \\
& \leq\left\|x_{n}-y_{n}\right\|+\left\|S y_{n}-z_{n}\right\|+\left\|z_{n}-x_{n}\right\| \\
& \rightarrow 0
\end{aligned}
$$

as $n \rightarrow \infty$. Therefore, by (9)-(10) and Lemma 3.2, we obtain

$$
\omega_{w}\left(x_{n}\right) \subset \operatorname{Fix}(T) \cap \operatorname{Fix}(S) .
$$

This, together with (4) and Lemma 3.4, guarantees the strong convergence of $\left\{x_{n}\right\}$ to $P_{\text {Fix }(T) \cap \operatorname{Fix}(S)} x_{0}$. This completes the proof.

Let $S=T$ in Algorithm 1, we have a hybrid algorithm for a nonexpansive mapping $T$ :

$$
\left\{\begin{array}{l}
x_{0} \in C \text { chosen arbitrarily, } \\
y_{n}=\alpha_{n} x_{n}+\left(1-\alpha_{n}\right) T x_{n}, \\
z_{n}=\beta_{n}\left[\gamma_{n} y_{n}+\left(1-\gamma_{n}\right) x_{n}\right]+\left(1-\beta_{n}\right) T y_{n}, \\
C_{n}=\left\{z \in C: \sigma\left\|z_{n}-z\right\|^{2}+(1-\sigma)\left\|y_{n}-z\right\|^{2} \leq\left\|x_{n}-z\right\|^{2}\right\} \\
Q_{n}=\left\{z \in C:\left\langle x_{n}-z, x_{n}-x_{0}\right\rangle \leq 0\right\} \\
x_{n+1}=P_{C_{n} \cap Q_{n}} x_{0}
\end{array}\right.
$$

for each $n \geq 0$, where $\alpha_{n}, \beta_{n} \in[0,1-\delta], \delta \in[0,1), \gamma_{n} \in[0,1], \sigma \in(0,1)$.

When $\gamma_{n}=0$, the algorithm (11) is actually a modification of the Ishikawa iteration ([24]). In 2006, Martinez-Yanes and Xu [23] modified the Ishikawa iteration and introduced the 
following algorithm:

$$
\left\{\begin{array}{l}
x_{0} \in C \text { chosen arbitrarily, } \\
y_{n}=\alpha_{n} x_{n}+\left(1-\alpha_{n}\right) T x_{n}, \\
z_{n}=\beta_{n} x_{n}+\left(1-\beta_{n}\right) T y_{n}, \\
C_{n}=\left\{z \in C:\left\|z_{n}-z\right\|^{2} \leq \beta_{n}\left\|x_{n}-z\right\|^{2}+\left(1-\beta_{n}\right)\left\|y_{n}-z\right\|^{2}\right\}, \\
Q_{n}=\left\{z \in C:\left\langle x_{n}-z, x_{n}-x_{0}\right\rangle \leq 0\right\}, \\
x_{n+1}=P_{C_{n} \cap Q_{n}} x_{0}
\end{array}\right.
$$

for each $n \geq 0$, where $\left\{\alpha_{n}\right\},\left\{\beta_{n}\right\}$ are the sequences in $[0,1]$ such that $\alpha_{n} \rightarrow 1$ and $\beta_{n} \leq 1-\delta$ for some $\delta \in(0,1]$.

It is easily observed that the algorithm (11) with $\gamma_{n}=0$ is different from the algorithm (12) in the definitions of the sets $C_{n}$ and the conditions on $\alpha_{n}$.

From Theorem 4.1, we get directly the following result.

Corollary 4.1 Let $C$ be a nonempty closed convex subset of a Hilbert space $H$ and $T: C \rightarrow$ $C$ be a nonexpansive mapping such that $\operatorname{Fix}(T) \neq \emptyset$. Assume that $\left\{\alpha_{n}\right\},\left\{\beta_{n}\right\}$, and $\left\{\gamma_{n}\right\}$ are the sequences in $[0,1]$ such that $\alpha_{n}, \beta_{n} \leq 1-\delta$ for some $\delta \in(0,1]$. Assume $\sigma \in(0,1)$. Then the sequence $\left\{x_{n}\right\}$ generated by the algorithm (11) converges in norm to $P_{\mathrm{Fix}(T)} x_{0}$.

Letting $\gamma_{n}=1$ in Algorithm 1, from Theorem 4.1, we obtain the following result.

Corollary 4.2 Let $C$ be a nonempty closed convex subset of a Hilbert space $H$ and T, S: $C \rightarrow C$ be two nonexpansive mappings such that $\operatorname{Fix}(T) \cap \operatorname{Fix}(S) \neq \emptyset$. Assume that $\left\{\alpha_{n}\right\}$ and $\left\{\beta_{n}\right\}$ are the sequences in $[0,1]$ such that $\alpha_{n}, \beta_{n} \leq 1-\delta$ for some $\delta \in(0,1]$. Assume $\sigma \in(0,1)$. Then the sequence $\left\{x_{n}\right\}$ generated by the following algorithm:

$$
\left\{\begin{array}{l}
x_{0} \in C \text { chosen arbitrarily, } \\
y_{n}=\alpha_{n} x_{n}+\left(1-\alpha_{n}\right) T x_{n}, \\
z_{n}=\beta_{n} y_{n}+\left(1-\beta_{n}\right) S y_{n}, \\
C_{n}=\left\{z \in C: \sigma\left\|z_{n}-z\right\|^{2}+(1-\sigma)\left\|y_{n}-z\right\|^{2} \leq\left\|x_{n}-z\right\|^{2}\right\}, \\
Q_{n}=\left\{z \in C:\left\langle x_{n}-z, x_{n}-x_{0}\right\rangle \leq 0\right\}, \\
x_{n+1}=P_{C_{n} \cap Q_{n}} x_{0} \quad \text { for each } n \geq 0
\end{array}\right.
$$

converges in norm to $P_{\mathrm{Fix}(T) \cap \mathrm{Fix}(S)} x_{0}$.

Let $\left\{T_{k}: C \rightarrow C, k=1,2, \ldots, N\right\}$ be a finite family of nonexpansive mappings such that $\bigcap_{k=1}^{N} \operatorname{Fix}\left(T_{k}\right) \neq \emptyset$. The algorithm (13) can be extended to a finite family of nonexpansive 
mappings as follows:

$$
\left\{\begin{array}{l}
x_{0} \in C \text { chosen arbitrarily, } \\
y_{n, 1}=\alpha_{n, 1} x_{n}+\left(1-\alpha_{n, 1}\right) T_{1} x_{n}, \\
y_{n, k}=\alpha_{n, k} y_{n, k-1}+\left(1-\alpha_{n, k}\right) T_{k} y_{n, k-1}, \\
C_{n}=\left\{z \in C: \sum_{k=1}^{N} \sigma_{k}\left\|y_{n, k}-z\right\|^{2} \leq\left\|x_{n}-z\right\|^{2}\right\}, \\
Q_{n}=\left\{z \in C:\left\langle x_{n}-z, x_{n}-x_{0}\right\rangle \leq 0\right\}, \\
x_{n+1}=P_{C_{n} \cap Q_{n} x_{0}}
\end{array}\right.
$$

for each $n \geq 0$ and $k=2, \ldots, N$, where $\alpha_{n, k} \in[0,1-\delta], \delta \in(0,1], \sigma_{k} \in(0,1)$ for each $k=$ $1,2, \ldots, N$, and $\sum_{k=1}^{N} \sigma_{k}=1$. Note that the algorithm (14) is different from the cyclic and parallel algorithms $[20,21]$ and other algorithms for finding a common fixed point of a finite family of nonexpansive mappings.

Extending Corollary 4.2 to a finite family of nonexpansive mappings, we can easily obtain the strong convergence for the algorithm (14), whose proof is similar to Theorem 4.1 and omitted here.

Corollary 4.3 Let $C$ be a nonempty closed convex subset of a Hilbert space $H$ and $\left\{T_{k}: C \rightarrow C, k=1,2, \ldots, N\right\}$ be a finite family of nonexpansive mappings such that $\bigcap_{k=1}^{N} \operatorname{Fix}\left(T_{k}\right) \neq \emptyset$. Assume that $\left\{\alpha_{n_{k}}\right\}$ is the sequence in $[0,1]$ such that $\alpha_{n_{k}} \leq 1-\delta$ for each $k=1,2, \ldots, N$ for some $\delta \in(0,1]$. Assume that $\sigma_{k} \in(0,1)$ for each $k=1,2, \ldots, N$, and $\sum_{k=1}^{N} \sigma_{k}=1$. Then the sequence $\left\{x_{n}\right\}$ generated by the algorithm (14) converges in norm to

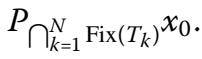

\section{Numerical experiments}

Many authors studied hybrid algorithms and analyzed strong convergence of the algorithms, however, as far as we know, the results of the realization for the algorithms are very limited (see, for example, $[25,26])$.

Recently, He et al. [27] pointed out that it is difficult to realize the hybrid algorithm in actual computing programs because the specific expression of $P_{C_{n} \cap Q_{n}} x_{0}$ cannot be got, in general. For the special case $C=H$, where $C_{n}$ and $Q_{n}$ are two half-spaces, they obtained the specific expression of $P_{C_{n} \cap Q_{n}} x_{0}$ and thus easily realized the hybrid algorithm proposed by Nakajo and Takahashi [13]. 
In the case $C=H$, following some ideas of He et al. [27], we obtain the specific expression of $P_{C_{n} \cap Q_{n}} x_{0}$ of Algorithm 1 as follows:

$$
\left\{\begin{array}{l}
x_{0} \in H \text { chosen arbitrarily, } \\
x_{n+1}=x_{n} \quad \text { if } T x_{n}=S x_{n}=x_{n} \\
y_{n}=\alpha_{n} x_{n}+\left(1-\alpha_{n}\right) T x_{n} \\
z_{n}=\beta_{n}\left[\gamma_{n} y_{n}+\left(1-\gamma_{n}\right) x_{n}\right]+\left(1-\beta_{n}\right) S y_{n}, \\
u_{n}=\sigma z_{n}+(1-\sigma) y_{n}-x_{n} \\
v_{n}=\frac{-\left(\sigma\left\|z_{n}-x_{n}\right\|^{2}+(1-\sigma)\left\|y_{n}-x_{n}\right\|^{2}\right)}{2} \\
C_{n}=\left\{z \in H:\left\langle u_{n}, x_{n}-z\right\rangle \leq v_{n}\right\} \\
Q_{n}=\left\{z \in H:\left\langle x_{n}-z, x_{n}-x_{0}\right\rangle \leq 0\right\} \\
x_{n+1}=p_{n}, \quad \text { if } p_{n} \in Q_{n} \\
x_{n+1}=q_{n}, \quad \text { if } p_{n} \notin Q_{n}
\end{array}\right.
$$

for each $n \geq 0$, where

$$
\begin{aligned}
& p_{n}=x_{0}-\frac{\left\langle u_{n}, x_{0}-x_{n}\right\rangle+v_{n}}{\left\|u_{n}\right\|^{2}} u_{n}, \\
& q_{n}=\left(1-\frac{\left\langle x_{0}-x_{n}, x_{n}-p_{n}\right\rangle}{\left\langle x_{0}-x_{n}, w_{n}-p_{n}\right\rangle}\right) p_{n}+\frac{\left\langle x_{0}-x_{n}, x_{n}-p_{n}\right\rangle}{\left\langle x_{0}-x_{n}, w_{n}-p_{n}\right\rangle} w_{n}, \\
& w_{n}=x_{n}-\frac{v_{n}}{\left\|u_{n}\right\|^{2}} u_{n} .
\end{aligned}
$$

Let $\mathbb{R}^{2}$ be a 2-dimensional Euclidean space with the usual inner product $\left\langle v^{(1)}, v^{(2)}\right\rangle=$ $v_{1}^{(1)} v_{1}^{(2)}+v_{2}^{(1)} v_{2}^{(2)}$ for all $v^{(1)}=\left(v_{1}^{(1)}, v_{2}^{(1)}\right)^{T}, v^{(2)}=\left(v_{1}^{(2)}, v_{2}^{(2)}\right)^{T} \in \mathbb{R}^{2}$, and the norm $\|v\|=\sqrt{v_{1}^{2}+v_{2}^{2}}$ $\left(v=\left(v_{1}, v_{2}\right)^{T} \in \mathbb{R}^{2}\right)$. He et al. [27] defined a mapping:

$$
T: v=\left(v_{1}, v_{2}\right)^{T} \mapsto\left(\sin \frac{v_{1}+v_{2}}{\sqrt{2}}, \cos \frac{v_{1}+v_{2}}{\sqrt{2}}\right)^{T}
$$

and showed that $T$ is nonexpansive. It is easily to observe that $T$ has a fixed point in the unit disk. Define a mapping $S: \mathbb{R}^{2} \rightarrow \mathbb{R}^{2}$ as follows:

$$
S(v):=P_{K}(v)
$$

for all $v \in \mathbb{R}^{2}$, where $K=\left\{v \in \mathbb{R}^{2}:\|v\| \leq 1\right\}$. It is well known that $S$ is nonexpansive (actually firmly nonexpansive) and $\operatorname{Fix}(S)=K$. Thus we get $\operatorname{Fix}(T) \cap \operatorname{Fix}(S)=\operatorname{Fix}(T) \neq \emptyset$.

Denote by $E(x)=\frac{\|x-T x\|+\|x-S x\|}{\|x\|}$ the relative rate of convergence of the algorithms since we do not know the exact value of the projection of $x_{0}$ onto common fixed points set of $S$ and $T$.

In the numerical results listed in the following tables, Iter. and Sec. denote the number of iterations and the cpu time in seconds, respectively. We took $E(x)<\varepsilon$ as the stopping criterion and $\varepsilon=10^{-4}$ unless specified otherwise. We chose different $x_{0}$ as initial point. The algorithms were coded in Matlab 7.1 and run on a personal computer.

We firstly investigated the choice of the parameters of Algorithm 1. 
Table 1 Algorithm 1 with $\alpha_{n}=0.1, \beta_{n}=0.1, \gamma_{n}=1.0$

\begin{tabular}{|c|c|c|c|c|c|c|c|c|c|c|}
\hline$x_{0}$ & $\sigma$ & 0.1 & 0.2 & 0.3 & 0.4 & 0.5 & 0.6 & 0.7 & 0.8 & 0.9 \\
\hline$(0,0)$ & Iter. & 372 & 472 & 404 & 538 & 539 & 417 & 461 & 452 & 523 \\
\hline$(2,7)$ & Iter. & 167 & 760 & 249 & 897 & 385 & 321 & 615 & 355 & 1,182 \\
\hline$(-5,2)$ & Iter. & 915 & 1,424 & 2,047 & 1,597 & 972 & 1,077 & 1,672 & 2,309 & 2,101 \\
\hline$(-3,-4)$ & Iter. & 1,032 & 669 & 1,930 & 1,166 & 934 & 766 & 753 & 1,181 & 530 \\
\hline
\end{tabular}

Table 2 Algorithm 1 with $\alpha_{n}=0.1, \beta_{n}=0.1, \sigma=0.1$

\begin{tabular}{lrrrrrrrrrrrr}
\hline $\boldsymbol{x}_{\mathbf{0}}$ & $\boldsymbol{\gamma}_{\boldsymbol{n}}$ & \multicolumn{1}{c}{$\mathbf{0 . 0}$} & \multicolumn{1}{c}{$\mathbf{0 . 1}$} & \multicolumn{1}{c}{$\mathbf{0 . 2}$} & \multicolumn{1}{c}{$\mathbf{0 . 3}$} & $\mathbf{0 . 4}$ & $\mathbf{0 . 5}$ & $\mathbf{0 . 6}$ & $\mathbf{0 . 7}$ & $\mathbf{0 . 8}$ & $\mathbf{0 . 9}$ & $\mathbf{1 . 0}$ \\
\hline$(0,0)$ & Iter. & 539 & 626 & 393 & 441 & 408 & 303 & 576 & 759 & 469 & 422 & 372 \\
$(2,7)$ & Iter. & 205 & 825 & 242 & 488 & 1,278 & 214 & 301 & 362 & 174 & 750 & 167 \\
$(-5,2)$ & Iter. & 1,237 & 1,373 & 1,853 & 1,627 & 2,275 & 2,173 & 1,609 & 2,036 & 1,316 & 2,633 & 915 \\
$(-3,-4)$ & Iter. & 919 & 780 & 1,647 & 958 & 875 & 957 & 1,411 & 663 & 1,146 & 573 & 1,032 \\
\hline
\end{tabular}

Table 3 Algorithm 1 with $\beta_{n}=0.1, \gamma_{n}=1.0, \sigma=0.1$

\begin{tabular}{|c|c|c|c|c|c|c|c|c|c|c|}
\hline$x_{0}$ & $\alpha_{n}$ & 0.1 & 0.2 & 0.3 & 0.4 & 0.5 & 0.6 & 0.7 & 0.8 & 0.9 \\
\hline$(0,0)$ & Iter. & 372 & 524 & 853 & 1,080 & 1,398 & - & - & - & - \\
\hline$(2,7)$ & Iter. & 167 & 482 & 625 & 1,479 & 2,186 & 2,046 & 3,115 & 4,111 & 14,440 \\
\hline$(-5,2)$ & Iter. & 915 & 1,418 & 2,950 & 3,998 & 6,098 & 6,827 & 1,330 & 20,114 & 68,421 \\
\hline$(-3,-4)$ & Iter. & 1,032 & 2,306 & 2,262 & 1,975 & 4,341 & 2,882 & 6,321 & 11,761 & 38,330 \\
\hline
\end{tabular}

In Algorithm 1, there are four parameters, $\sigma, \alpha_{n}, \beta_{n}, \gamma_{n}$ (indeed three nonnegative sequences). Thus we need to set them before performing the algorithm.

Tables 1 and 2 show the effect of different choice of $\sigma$ and $\gamma_{n}$ and illustrated that the number of iteration was relatively small as $\sigma=0.1$ and $\gamma_{n}=1.0$ for the initial points $(0,0)$, $(2,7)$, and $(-5,2)$.

Table 3 lists the impact of $\alpha_{n}$ on the efficiency of the algorithm and showed that the number of iteration was increasing with $\alpha_{n}$, with few exceptions. For $\alpha_{n} \geq 0.6$, the stopping criterion could not even be satisfied at initial point $(0,0)$. The impact of $\beta_{n}$ was similar to that of $\alpha_{n}$ and we do not list it here.

Next, we discuss the choice of parameters in Algorithms 2 and 3. We took $\varepsilon=5 \times 10^{-4}$, since for Algorithms 2 and 3, it was difficult for the relative rate of convergence to run up to $10^{-4}$. For Algorithm 2, we tested $\alpha_{n}=[0.1,0.9]$ and the numerical results are reported in Figure 1, which showed that the number of iterations was less for small $\alpha_{n}$. Since the number of iterations for $\alpha_{n}>0.6$ was generally larger than those for $\alpha_{n} \leq 0.6$, we only report the results for $0.1 \leq \alpha_{n} \leq 0.6$. For Algorithm 3, Figure 2 reports the number of iterations for $0.1 \leq \alpha_{n} \leq 0.6$, where $\beta_{n}=\gamma_{n}=\left(1-\alpha_{n}\right) / 2$. The conclusion was similar to Algorithm 3, that is, small $\alpha_{n}$ was better.

Finally, we compare Algorithm 1 with Algorithms 2 and 3. We took $\alpha_{n}=0.1, \beta_{n}=0.1$, $\gamma_{n}=1.0, \sigma=0.1$ for Algorithm 1, $\alpha_{n}=0.1$ in Algorithm 2 and $\alpha_{n}=0.1, \beta_{n}=\gamma_{n}=0.45$ in Algorithm 3. The stopping criterion was $E(x)<\varepsilon=5 \times 10^{-5}$. Table 4 illustrates the efficiency of the Algorithm 1, both from the points of view of number of iterations and cpu time.

\section{Conclusions}

Hybrid algorithms for nonexpansive mappings have extensively been studied over the past decade. In this paper, we introduced a new hybrid algorithm and, for the first time in the 


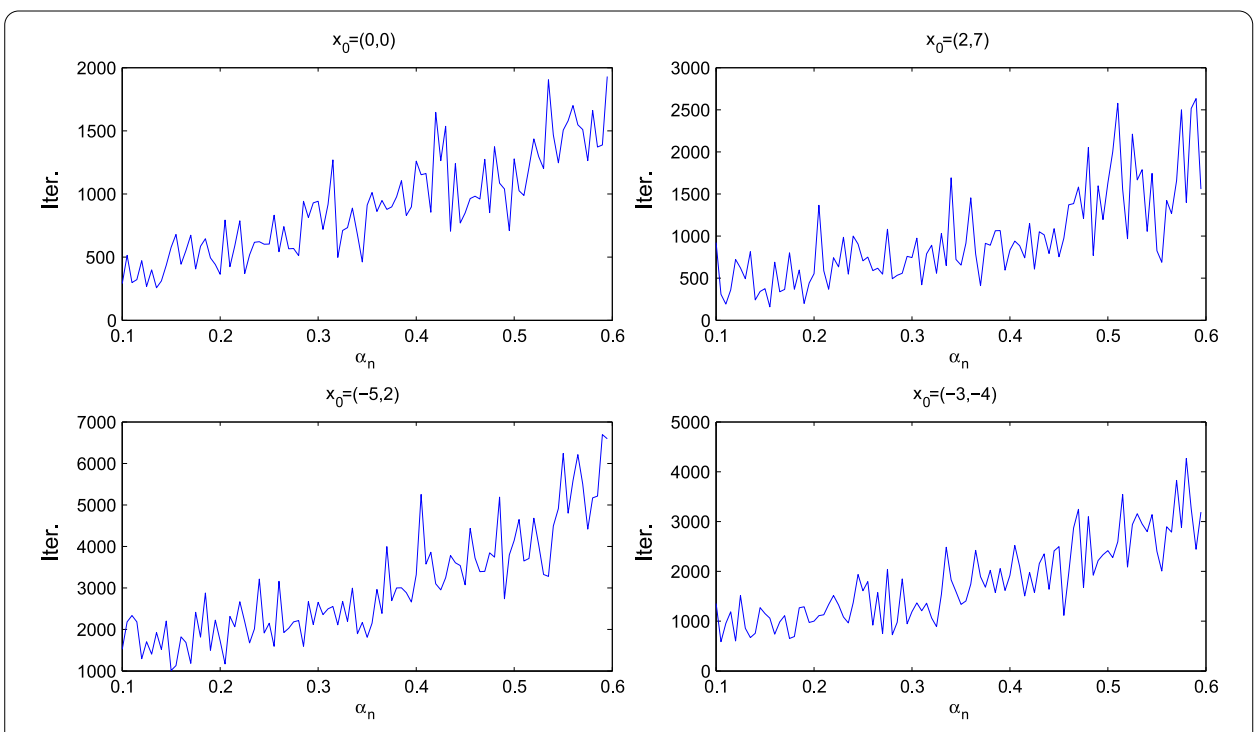

Figure 1 Comparison of the number of iterations of Algorithm 2 with different initial values.

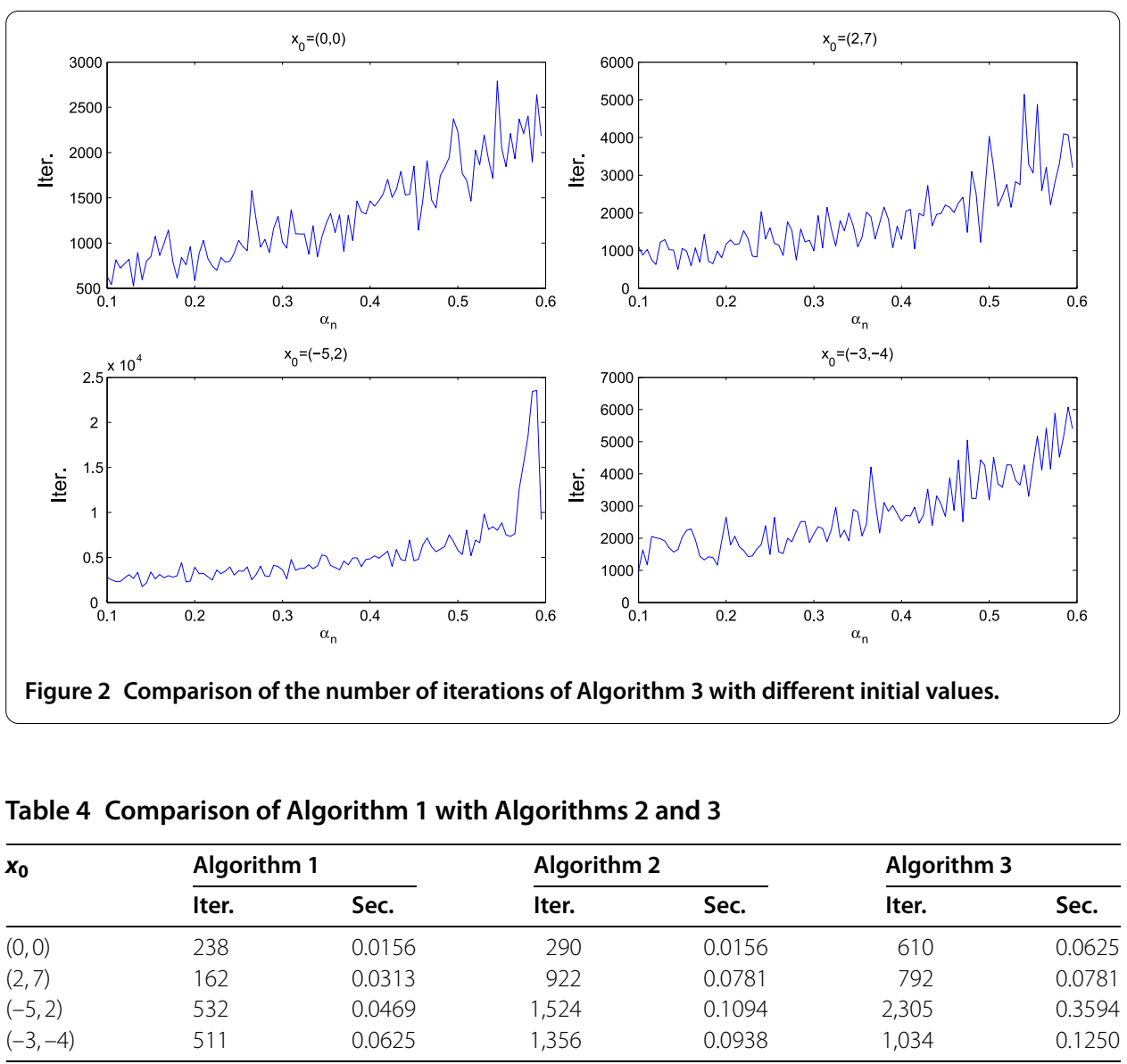

literature, compared the different hybrid algorithms in computing. Numerical examples were provided, which showed the advantages of the new algorithm. 


\section{Competing interests}

The authors declare that they have no competing interests.

\section{Authors' contributions}

All authors contributed equally to the writing of this paper. All authors read and approved the manuscript.

\section{Author details}

${ }^{1}$ College of Science, Civil Aviation University of China, Tianjin, 300300, China. ${ }^{2}$ Tianjin Key Lab for Advanced Signal Processing, Civil Aviation University of China, Tianjin, 300300, China. ${ }^{3}$ Department of Mathematics Education and RINS, Gyeongsang National University, Jinju, 660-701, Korea. ${ }^{4}$ Department of Mathematics, Faculty of Science, King Abdulaziz University, Jeddah, Saudi Arabia.

\section{Acknowledgements}

The authors would like to express their thanks to Professor Peichao Duan for her help in program. The first and second authors were supported by National Natural Science Foundation of China (No. 11201476) and Fundamental Research Funds for the Central Universities (No. 3122013D017), in part by the Foundation of Tianjin Key Lab for Advanced Signal Processing and the third author was supported by Basic Science Research Program through the National Research Foundation of Korea (NRF) funded by the Ministry of Science, ICT and future Planning (2014R1A2A2A01002100).

\section{Received: 30 December 2014 Accepted: 7 August 2015 Published online: 22 August 2015}

\section{References}

1. Chang, SS: Viscosity approximation methods for a finite family of nonexpansive mappings in Banach spaces. J. Math. Anal. Appl. 323, 1402-1416 (2006)

2. Atsushiba, S, Takahashi, W: Strong convergence theorems for a finite family of nonexpansive mappings and applications. Indian J. Math., 41(3), 435-453 (1999). BN Prasad birth centenary commemoration volume.

3. Yao, Y: A general iterative method for a finite family of nonexpansive mappings. Nonlinear Anal. 66, 2676-2687 (2007)

4. Xu, HK: A variable Krasnosel'skiĭ-Mann algorithm and the multiple-set split feasibility problem. Inverse Probl. 22, 2021-2034 (2006)

5. Combettes, PL: On the numerical robustness of the parallel projection method in signal synthesis. IEEE Signal Process. Lett. 8, 45-47 (2001)

6. Podilchuk, Cl, Mammone, RJ: Image recovery by convex projections using a least-squares constraint. J. Opt. Soc. Am. 7, 517-521 (1990)

7. Youla, D: Mathematical theory of image restoration by the method of convex projection. In: Stark, $\mathrm{H}$ (ed.) Image Recovery Theory and Applications, pp. 29-77. Academic Press, Orlando (1987)

8. Mann, WR: Mean value methods in iteration. Proc. Am. Math. Soc. 4, 506-510 (1953)

9. Genel, A, Lindenstrass, J: An example concerning fixed points. Isr. J. Math. 22, 81-86 (1975)

10. Bauschke, $\mathrm{HH}$ : The approximation of fixed points of compositions of nonexpansive mappings in Banach spaces J. Math. Anal. Appl. 202, 150-159 (1996)

11. Wang, F: Iterative methods for nonlinear optimization problems in Hilbert spaces (in Chinese), Ph.D. thesis, East China University of Science and Technology (2011).

12. Solodov, MV, Svaiter, BF: Forcing strong convergence of proximal point iterations in a Hilbert space. Math. Program., Ser. A 87, 189-202 (2000)

13. Nakajo, K, Takahashi, W: Strong convergence theorems for nonexpansive mappings and nonexpansive semigroups. J. Math. Anal. Appl. 279, 372-379 (2003)

14. Kim, TH, Xu, HK: Strong convergence of modified Mann iterations. Nonlinear Anal. 61, 51-60 (2005)

15. Marino, G, Xu, HK: Weak and strong convergence theorems for strict pseudo-contractions in Hilbert spaces. J. Math. Anal. Appl. 329, 336-346 (2007)

16. Wei, L, Cho, YJ, Zhou, HY: A strong convergence theorem for common fixed points of two relatively nonexpansive mappings and its applications. J. Appl. Math. Comput. 29, 95-103 (2009)

17. Zhou, H, Su, Y: Strong convergence theorems for a family of quasi-asymptotic pseudo-contractions in Hilbert spaces. Nonlinear Anal. 70, 4047-4052 (2009)

18. Nilsrakoo, W, Saejung, S: Weak and strong convergence theorems for countable Lipschitzian mappings and its applications. Nonlinear Anal. 69, 2695-2708 (2008)

19. Eckstein, J, Svaiter, BF: A family of projective splitting methods for the sum of two maximal monotone operators. Math. Program., Ser. B 111, 173-199 (2008)

20. Takahashi, W, Takeuchi, Y, Kubota, R: Strong convergence theorems by hybrid methods for families of nonexpansive mappings in Hilbert spaces. J. Math. Anal. Appl. 341, 276-286 (2008)

21. Plubtieng, S, Ungchittrakool, K: Strong convergence theorems for a common fixed point of two relatively nonexpansive mappings in a Banach space. J. Approx. Theory 149, 103-115 (2007)

22. Goebel, K, Kirk, WA: Topics in Metric Fixed Point Theory. Cambridge Studies in Advanced Mathematics, vol. 28. Cambridge University Press, Cambridge (1990)

23. Martinez-Yanes, C, Xu, HK: Strong convergence of the CQ method for fixed point processes. Nonlinear Anal. 64, 2400-2411 (2006)

24. Ishikawa, S: Fixed points by a new iteration method. Proc. Am. Math. Soc. 44, 147-150 (1974)

25. Dong, QL, Lu, YY: A new hybrid algorithm for a nonexpansive mapping. Fixed Point Theory Appl. 2015, 37 (2015)

26. He, S, Yang, Z: Realization-based method of successive projection for nonexpansive mappings and nonexpansive semigroups (submitted)

27. He, S, Yang, C, Duan, P: Realization of the hybrid method for Mann iterations. Appl. Math. Comput. 217, 4239-4247 (2010) 